\title{
Empirical Relations about the Number of Dimensions in Theoretical Physics with the Concept of Common and Unshared Dimensions
}

\section{Tomofumi Miyashita}

Miyashita Clinic Mitsuya-Kita, Osaka, Japan

Email: tom_miya@plala.or.jp

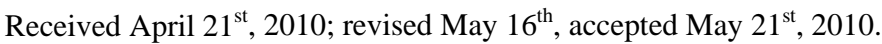

\begin{abstract}
How many dimensions are there in the universe? Currently, there is confusion about the number of dimensions in the universe. Empirical relations about the number of dimensions in theoretical physics with the concept of common spacetime 4 dimensions and unshared dimensions are described in this report.
\end{abstract}

Keywords: Kaluza-Klein, Superstring, Super gravity, Nambu String, Unshared Dimensions

\section{Introduction}

Einstein discovered space-time 4 dimensions. In order to complete the theory of everything (TOE), the numbers of dimensions have been increased. Currently, there is confusion about the number of dimensions in the universe. There is a simple question, "How many dimensions are there in the universe?"

The concept of "unshared dimensions" is very suitable solution. Unshared dimensions belong to each particle and the numbers of unshared dimensions are different between the different kinds of particles.

This paper presents empirical relations about the number of dimensions in theoretical physics with the concept of common space-time 4 dimensions and unshared dimensions.

\section{Empirical Relations about the Number of Dimensions in Theoretical Physics}

\subsection{The Concept of Unshared Dimension}

There are two kinds of dimensions. One is common spacetime 4 dimensions and the other is unshared dimensions which belong to each particle. The common space-time 4 dimensions are entirely same dimensions which Einstein discovered.

Unshared dimensions are something internal dimensions in each particle. The concept of unshared dimensions is discovered empirically. So, the mathematical explanation of "unshared dimensions" is the next stage argument. It is important that even the space has one unshared dimension. So, the definition of the space is different from the common space-time 4 dimensions.

\subsection{Empirical Relations about the Number of Dimensions in Theoretical Physics}

The number of unshared dimensions can be expressed empirically as:

Unshared dimension $=4 \times(4-\mathrm{N}+1) / \mathrm{N}$

$\mathrm{N}$ is the number related with the symmetry. Calculated unshared dimensions are shown in Table 1. Empirical relations about the number of dimensions in theoretical physics are shown in Table 2.

When $\mathrm{N}=3$, then unshared dimensions are $8 / 3$. In this case, explaining for quarks is difficult. 8 and $1 / 3$ is related with a quark, because the number of gluon is 8 and a quark must exist with three particles.

Simple evidences supported unshared dimensions can be shown in the next section.

\section{The Evidence of Unshared Dimensions}

\subsection{Empirical Relations between the Masses of Leptons}

If leptons have 6 unshared dimensions, the mass of leptons are expected like this.

$$
M=k \times \int x^{6} d x=\frac{k}{7} x^{7}
$$

Here, $\mathrm{M}, \mathrm{k}$ and $\mathrm{x}$ are the mass of lepton, constant coefficient and the value of unshared dimensions. 


$$
y=\left(\frac{M}{m}\right)^{\frac{1}{7}}
$$

Here, $m$ and $y$ are the mass of electron and the seventh power root of ratio with electron. The calculation results are shown in Table 3.The square of correlation coefficient between the generation and the seventh power root of mass ratio is 0.9996 shown in Figure 1. It is impossible that this result is only coincidence. The expected mass of the forth lepton and the fifth lepton are $14.3 \mathrm{GeV}$ and $70.5 \mathrm{GeV}$ respectively. The peak of $14.3 \mathrm{eV}$ was reported [1] and there remains the possibility of the forth lepton.

The expected leptons which have larger generation number should be very unstable, since they cannot be discovered still yet. But there are many experimental reports about the reactions around the expected energy. The reports published in the internet are much more than those around the unexpected energy.

\subsection{The Mass of Weak Boson}

Weak boson is related with electrons and neutrino. Then, the number of unshared dimension is $26(=10+20-4)$. The 27th power root of ratio between Weak boson and electron are shown in Table 4. The result is near the generation number 2 . If the generation number is 3 , the mass of Weak boson is $4 \times 10^{6} \mathrm{TeV}$ and it is impossible to observe.

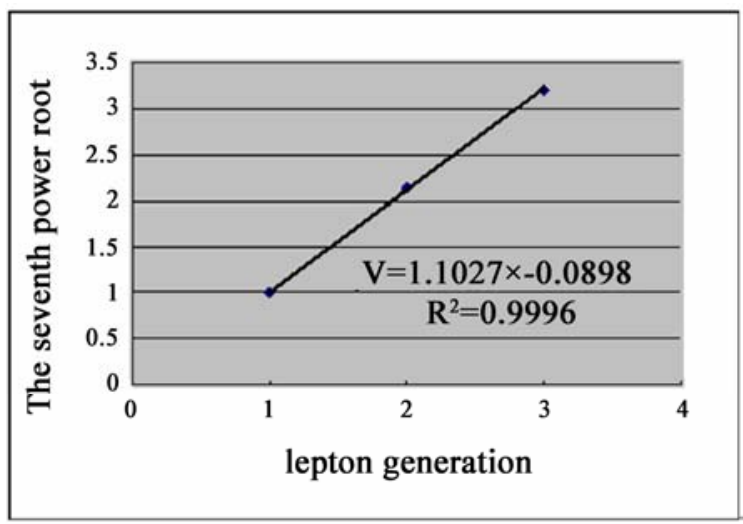

Figure 1. Empirical Relation about the mass of lepton

Table 1. Calculated unshared dimensions

\begin{tabular}{cccccc}
\hline $\mathrm{N}$ & 4 & 3 & 2 & 1 & $(0)$ \\
\hline particle & The space & quark & lepton & neutrino & consciousness \\
Unshared dimension & 1 & $8 / 3$ & 6 & 16 & infinite \\
\hline
\end{tabular}

Table 2. Empirical relations about the number of dimensions in theoretical physics

\begin{tabular}{|c|c|c|c|c|c|c|}
\hline Theory & common & The space & quark & lepton & neutrino & total \\
\hline Kaluza-Klein & 4 & 1 & - & - & - & 5 \\
\hline $\begin{array}{c}\text { Superstring } \\
(\sim 1980 \mathrm{~s})\end{array}$ & 4 & - & - & 6 & - & 10 \\
\hline Nambu string & 4 & - & - & 6 & 16 & 26 \\
\hline Superstring ( 2000s) & 4 & 1 & - & 6 & 16 & 27 \\
\hline
\end{tabular}

Table 3. The seventh power root

\begin{tabular}{ccccc}
\hline & Generation & mass $(\mathrm{MeV})$ & Mass ratio & The seventh power root of mass ratio \\
\hline electron & 1 & 0.51099906 & 1 & 1 \\
Muon & 2 & 105.6 & 206.6540005 & 2.141653053 \\
Tauon & 3 & 1776.99 & 3477.481935 & 3.20549411 \\
4th & 4 & 14371.76031 & 28124.82729 & 4.321 \\
5th & 5 & 70548.8140 & 138060.5555 & 5.424 \\
6th & 6 & 257713 & 504331 & 6.526 \\
7th & 7 & 768658 & 1504226 & 7.629 \\
8th & 8 & 1977637 & 3870138 & 8.732 \\
\hline
\end{tabular}



with the Concept of Common and Unshared Dimensions

Table 4. The 27th power root

\begin{tabular}{ccccc}
\hline & Generation & mass $(\mathrm{MeV})$ & Mass ratio & The 27th power root of mass ratio \\
\hline electron & 1 & 0.51099906 & 1 & 1 \\
W-boson & 2 & 80398 & 157334.9274 & 2.021601712 \\
Z-boson & 2 & 91187.6 & 178449.6433 & 2.036632582 \\
\hline
\end{tabular}

\section{Summary}

The concept of common space-time 4 dimensions and unshared dimensions are discovered empirically. Simple evidences supported unshared dimensions can be shown from the mass of particles. It is very useful concept to answer for the question, "How many dimensions are there in the universe?”

\section{REFERENCES}

[1] C. Friberg, E. Norrbin, T. Sjöstrand, "QCD Aspects of Leptoquark Production at HERA," Physics Letter B, Vol. 403, No. 3-4, June 1997, pp. 329-334. 